\title{
Pharmacodynamic differences of rocuronium in immobilized ICU patients
}

\section{E. Brands, ETZ, Dept of Intensive Care, Tilburg, Netherlands}

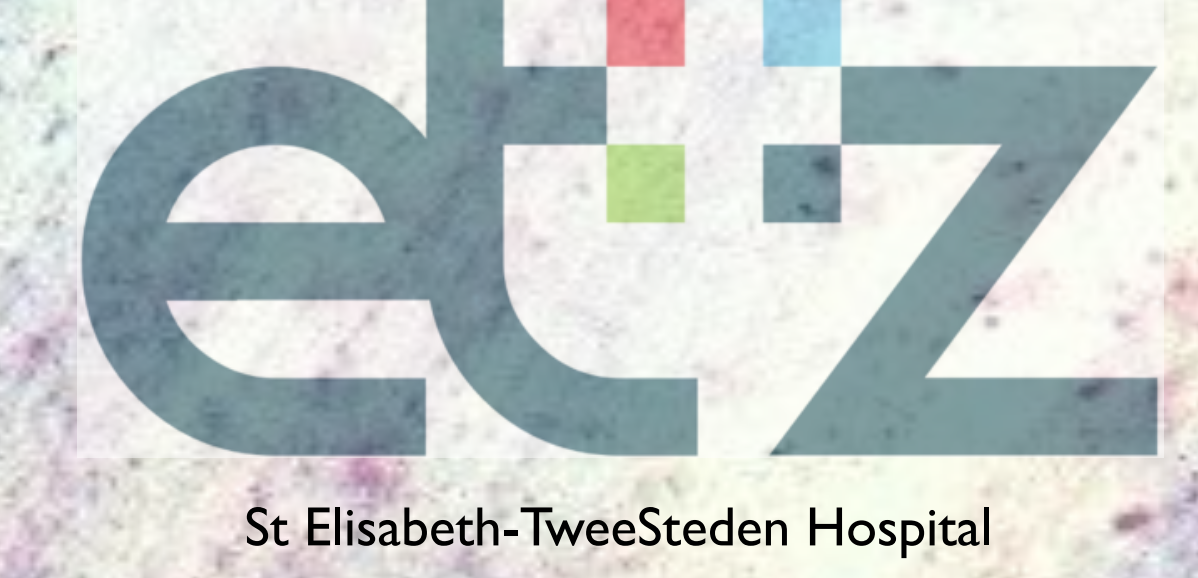

\section{Background:}

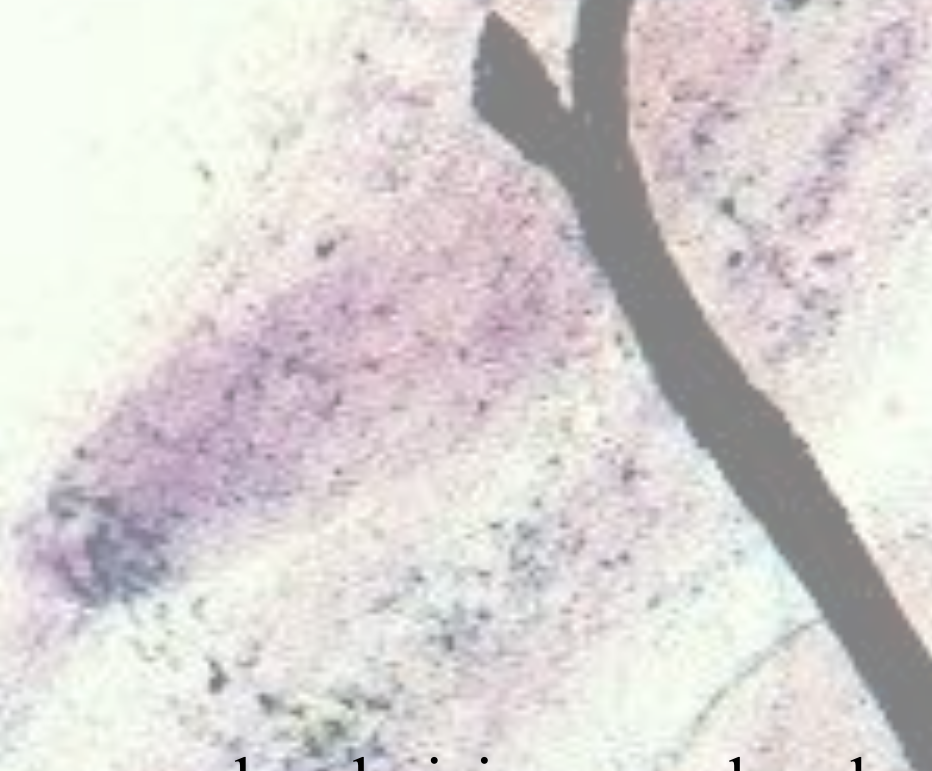

Rocuronium is a non-depolarizing muscle relaxant with an onset time of $45-60$ seconds when using $1.2 \mathrm{mg} / \mathrm{kg}$. It has replaced succinylcholine for rapid sequence $<$ induction (RSI) especially when there is a contraindication for succinylcholine, for instance hyperkalemia due to up-regulation of immature Acetylcholine receptors (AchRs) during prolonged immobilization. We noticed a decreased sensitivity to rocuronium in immobilized patients during placement of a percutaneous dilatational tracheostomy (PDT) en decided to test this.

\section{Materials and methods:}

- 20 ICU patients in need for a PDT

- Excluded: tetraplegíc or CIPNP patients

- $1.2 \mathrm{mg} / \mathrm{kg}$ rocuronium

- Onset time measurement using a TOF-watch

- Relation onset time - time to decrease to 1 twitch (3 patients, being immobilized for more than 20 days, didn't achieve a full block with zero twitches) (I)

Results:

- Spearman correlation onset $-\mathrm{T}$ down to 1 twitch $=$ 595 (p .006). Moderate to strong correlation (fig 1)

- Significantly different onset time (KW .006) when grouping patients in immobilization days $\leq 7,8-14$ and $\geq 15$ days

MW-U group $1-2$ p 0.026 , group $1=3$ p 0.008

No differences in age, BMI, Apache II, sofa, edema, temperature, $\mathrm{pH}$ and interacting co-medication except for sedation 12 hours prior to the PDT (KW p .012).

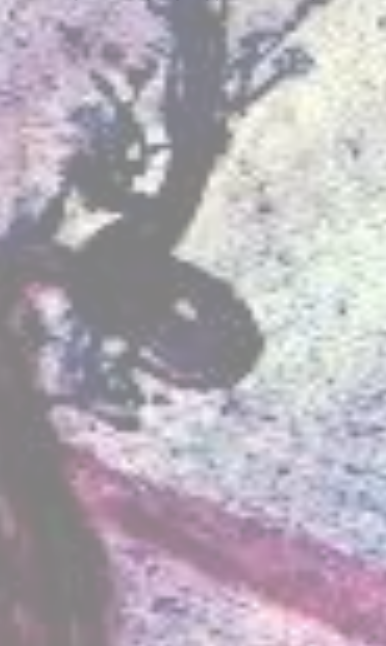

References:

1. Greenberg ea. The use of neuromuscular blocking agents in the ICU. Crit Care Med 2013; 41: 1332-44

2. Blanié ea. The limits of succinylcholine for critically ill patients Anesth Analg 2012; 115:873-9

\section{Discussion:}

Immobilized patients seem to develop a decreased sensitivity to rocuronium with prolonged onset time. We believe the effect of up-regulation of immature AchRs outweighs the effect of muscle wasting during immobilization. This can have implications when a fast deep neuromuscular block is needed in this patient group.

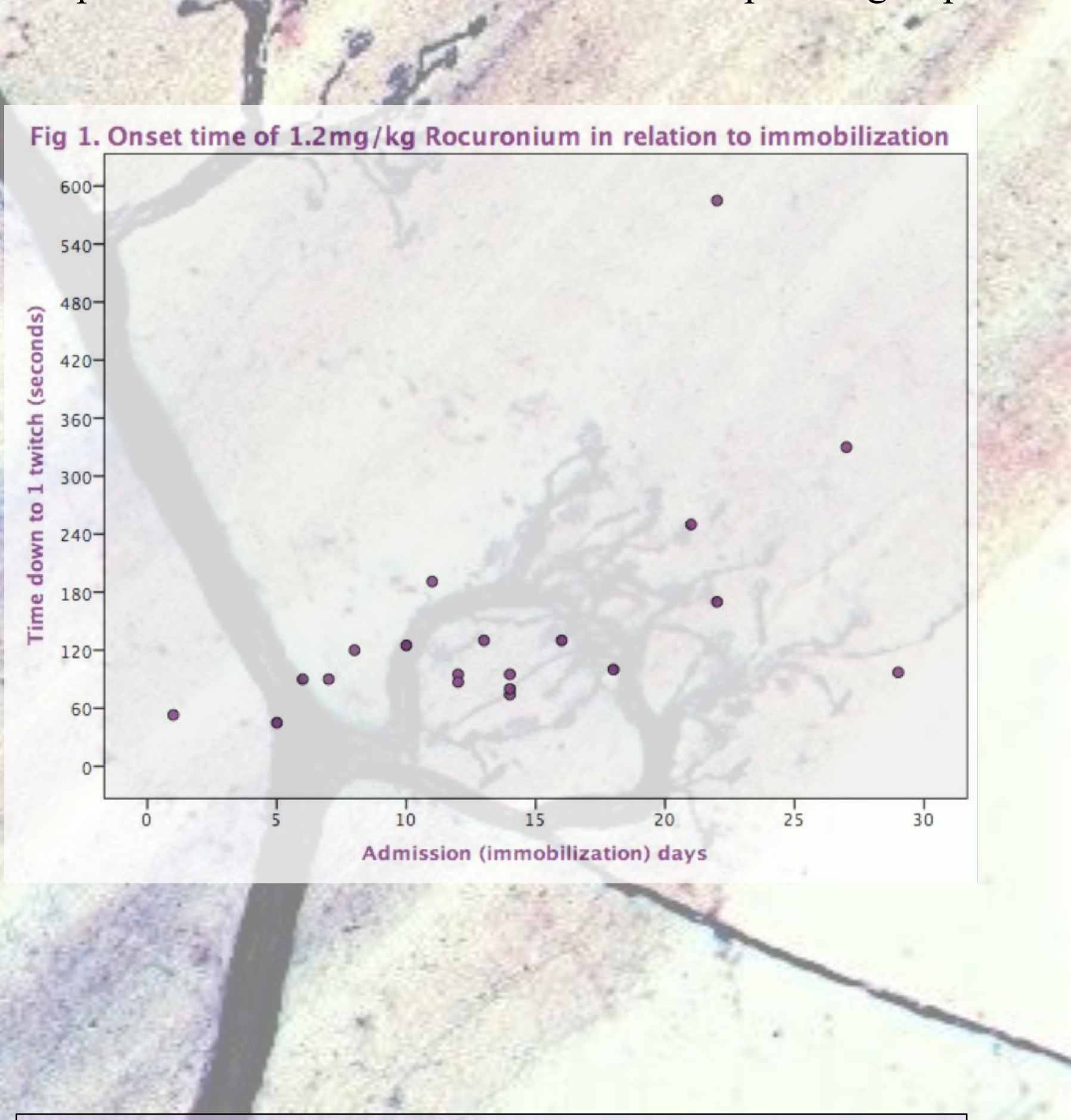

\section{Conclusion(s):}

When using rocuronium for RSI in immobilized patients one should consider a prolonged onset time and consider using a higher dosage than usual when aspiration risk is high. 